\title{
Interference Cancellation for MIMO Systems Employing the Generalized Receiver with High Spectral Efficiency
}

\author{
VYACHESLAV TUZLUKOV, \\ Belarusian State Aviation Academy \\ 77, Uborevicha Str., 220096 Minsk \\ BELARUS
}

\begin{abstract}
In this paper, we investigate the performan-ce in terms of symbol error probability (SEP) of multipleinput multiple-output (MIMO) systems employing the ge-neralized receiver with high spectral efficiency. In particular, we consider the coherent detection of $M$-PSK signals in a flat Rayleigh fading environment. We focus on spectrally efficient MIMO systems where after serial-to-parallel con-version, several sub-streams of symbols are simultaneously transmitted by using an antenna array, thereby increasing the spectral efficiency. The reception is based on linear mi-nimum mean-square-error (MMSE) combining, eventually followed by successive interference cancellation. Exact and approximate expressions are derived for an arbitrary nu-mber of transmitting and receiving antenna elements. Sim-ulation results confirm the validity of our analytical meth-odology.
\end{abstract}

Keywords-Error propagation, minimum mean-square-error (MMSE) methods, multiple-input multiple-output (MIMO) systems, generalized receiver, Rayleigh channels, interference cancellation.

Received: July 18, 2020. Revised: January 5, 2021. Accepted: January 27, 2021. Published: January 31, 2021.

\section{Introduction}

$\mathrm{O}$

VER the last several decades, multiple antennas have been used to combat fast fading. Increase in diversity order provided by diversity techniques enables robust communications in a fading environment [1], [2]. More recently, it has been recognized that the capacity of wireless communication links is increased by using multiple antennas both at the transmitter and the receiver [3], [4]. Toward achieving these capacities, a promising transmission system, called Diagonal-Bell Laboratories Layered Space-Time (D-BLAST), has been proposed in [3]. This scheme is able to provide a high spectral efficiency in a rich and quasistatic scattering environment. Owing to the large computational complexity required for this scheme, a simplified version, called Vertical BLAST (VBLAST) has been proposed in [5]. A BLAST scheme is primarily based on the following three steps:

- interference nulling to reduce the effect of the other (interfering) signals on the desired one;

- $\quad$ ordering to select the sub-stream with the largest signal-to-noise ratio $(S N R)$;

- $\quad$ successive interference cancellation (SIC).

Moreover, it was observed also in [3] that a minimum meansquare-error (MMSE) criterion can be used instead of interference nulling (zero forcing) to mitigate both interference and thermal noise.

Some papers [4], [6]-[8] propose an analytical evaluation of the capacity of MIMO systems. Some other papers address simulation of MIMO systems in frequency flat or selective fading channels [9]-[11], while in [12] the investigation of capability of V-BLAST systems with maximum likelihood detec- tion to reduce interference and thermal noise contributions has been carried out. In the present paper, we investigate the performance of multiple-input multiple-output (MIMO) systems employing the generalized receiver with high spectral efficiency. In particular, we consider the symbol error probability (SEP) for coherent detection of $M$-ary phase shift keying (PSK) signals in a flat Rayleigh-fading environment.

We start from the analytical framework developed in [13] for optimum combining (OC) of signals in multi-antenna systems in the presence of co-channel interferers and thermal noise. This framework enables us to investigate the performance of MIMO-MMSE systems employing the generalized receiver in flat Rayleigh fading channels. We generalize this methodlogy to derive the SEP for MIMO-MMSE system followed by SIC. We refer to this system as MIMO-MMSE-SIC and first investigate its performance for the cases of no error propagation (EP). We then extend our study to include the effects of EP.

The paper is organized as follows. In Section II, we provide the generalized receiver description. In Section III, we present the system description and the basic mathematics that is necessary for understanding the MIMO-MMSE generalized receiver. In Section IV, we give a new expression for analyzing optimum combining of signals and derive the SEP at the MIMOMMSE generalized receiver output. In Section V, we present the performance analysis of MIMO-MMSE system employing the generalized receiver with SIC. Finally, in Section VI, we demonstrate some numerical results, including the comparison with simulation. Conclusions are given in Section VII. 


\section{Generalized Receiver}

The generalized receiver is constructed in accordance with the generalized approach to signal processing in noise [14][16]. The generalized approach to signal processing in noise introduces an additional noise source that does not carry any information about the parameters of desired transmitted signal with the purpose to improve the signal processing system performance. This additional noise can be considered as the reference noise without any information about the parameters of the signal to be detected.

The jointly sufficient statistics of the mean and variance of the likelihood function is obtained under the generalized approach to signal processing in noise employment, while the classical and modern signal processing theories can deliver only a sufficient statistics of the mean or variance of the likelihood function. Thus, the generalized approach to signal processing in noise implementation allows us to obtain more information about the parameters of the desired transmitted signal incoming at the generalized receiver input. Owing to this fact, the detectors constructed based on the generalized approach to signal processing in noise technology are able to improve the signal detection performance of signal processing systems in comparison with employment of other conventional detectors.

The generalized receiver (GR) consists of three channels (see Fig.1): the GR correlation detector (GR CD) channel the preliminary filter $(\mathrm{PF})$, the multipliers 1 and 2, the model signal generator (MSG); the GR energy detector (GR ED) channel - the PF, the additional filter (AF), the multipliers 3 and 4, the summator 1; and the GR compensation channel (GR CC) - the summators 2 and 3, the accumulator 1. The threshold apparatus (THRA) device defines the GR threshold. As we can see from Fig.1, there are two band-pass filters, i.e. the linear systems, at the GR input, namely, the PF and AF. We assume for simplicity that these two filters or linear systems have the same amplitude-frequency characteristics or impulse responses. The AF central frequency is detuned relative to the $\mathrm{PF}$ central frequency.

There is a need to note that the PF bandwidth is matched with the transmitted signal bandwidth. If the detuning value betwe-en the PF and AF central frequencies is more than 4 or 5 times the transmitted signal bandwidth to be detected, i.e., where there is the transmitted signal bandwidth, we can believe that the processes at the PF and AF outputs are uncorrelated because the coefficient of correlation between them is negligible (not more than 0.05). This fact was confirmed experimentally in [17] and [18] independently. Thus, the transmitted signal plus noise can be appeared at the GR PF output and the noise only is appeared at the GR AF output. The stochastic process-es at the GR AF and GR PF outputs present the input stochas-tic samples from two independent frequency-time regions. If the discrete-time noise at the GR PF and GR AF inputs is Gaussian, the discrete-time noise at the GR PF output is Gaussian too, and the reference discrete-time noise at the GR AF output is Gaussian owing to the fact that the GR PF and GR $\mathrm{AF}$ are the linear systems and we believe that these linear systems do not change the statistical parameters of the input pro- cess. Thus, the GR AF can be considered as a generator of the reference noise with a priori information a "no" transmitted signal (the reference noise sample) [16, Chapter 5]. The noise at the GR PF and GR AF outputs can be presented as

$$
\left\{\begin{array}{l}
\zeta_{i}[k]=\sum_{m=-\infty}^{\infty} g_{P F}[m] w_{i}[k-m] ; \\
\eta_{i}[k]=\sum_{m=-\infty}^{\infty} g_{A F}[m] w_{i}[k-m],
\end{array}\right.
$$

where $g_{P F}[m]$ and $g_{A F}[m]$ are the impulse responses of the GR PF and GR AF, respectively.

In a general, under practical implementation of any detector in wireless communication system with sensor array, the bandwidth of the spectrum to be sensed is defined. Thus, the GR AF bandwidth and central frequency can be assigned, too (this bandwidth cannot be used by the transmitted signal because it is out of its spectrum). The case when there are interfering signals within the GR AF bandwidth, the action of this interference on the GR detection performance, and the case of non-ideal condition when the noise at the GR PF and GR AF outputs is not the same by statistical parameters are discussed in [19] and [20].

Under the hypothesis $\mathscr{H}_{1}$ ("a yes" transmitted signal), the GR CD generates the signal component $s_{i}^{m}[k] s_{i}[k]$ caused by interaction between the model signal $s_{i}^{m}[k]$, forming at the MSG output, and the incoming signal $s_{i}[k]$, and the noise component $s_{i}^{m}[k] \zeta_{i}[k]$ caused by interaction between the model signal $s_{i}^{m}[k]$ and the noise $\zeta_{i}[k]$ at the PF output. GR ED generates the transmitted signal energy $s_{i}^{2}[k]$ and the random component $s_{i}[k] \zeta_{i}[k]$ caused by interaction between the transmitted signal $s_{i}[k]$ and the noise $\zeta_{i}[k]$ at the PF output. The main purpose of the GR CC is to cancel completely in the statistical sense the GR CD noise component $s_{i}^{m}[k] \zeta_{i}[k]$ and the GR ED random component $s_{i}[k] \zeta_{i}[k]$ based on the same nature of the noise $\zeta_{i}[k]$. The relation between the transmitted signal to be detected $s_{i}[k]$ and the model signal $s_{i}^{m}[k]$ is defined as:

$s_{i}^{m}[k]=\mu s_{i}[k]$,

where $\mu$ is the coefficient of proportionality.

The main functioning condition under the GR employment in any signal processing system including the communication one with radar sensors is the equality between the parameters of the model signal $s_{i}^{m}[k]$ and the incoming signal $s_{i}[k]$, for example, by amplitude. Under this condition it is possible to cancel completely in the statistical sense the noise component $s_{i}^{m}[k] \zeta_{i}[k]$ of the GR CD and the random component $s_{i}[k] \times$ $\zeta_{i}[k]$ of the GR ED. Satisfying the GR main functioning condition given by (2), $s_{i}^{m}[k]=s_{i}[k], \mu=1$, we are able to detect the transmitted signal with the high probability of detection at 
the low $S N R$ and define the transmitted signal parameters with high accuracy.
Practical realization of this condition (2) at $\mu \rightarrow 1$ requires increasing in the complexity of GR structure and, consequently, leads us to increasing in computation cost. For example,

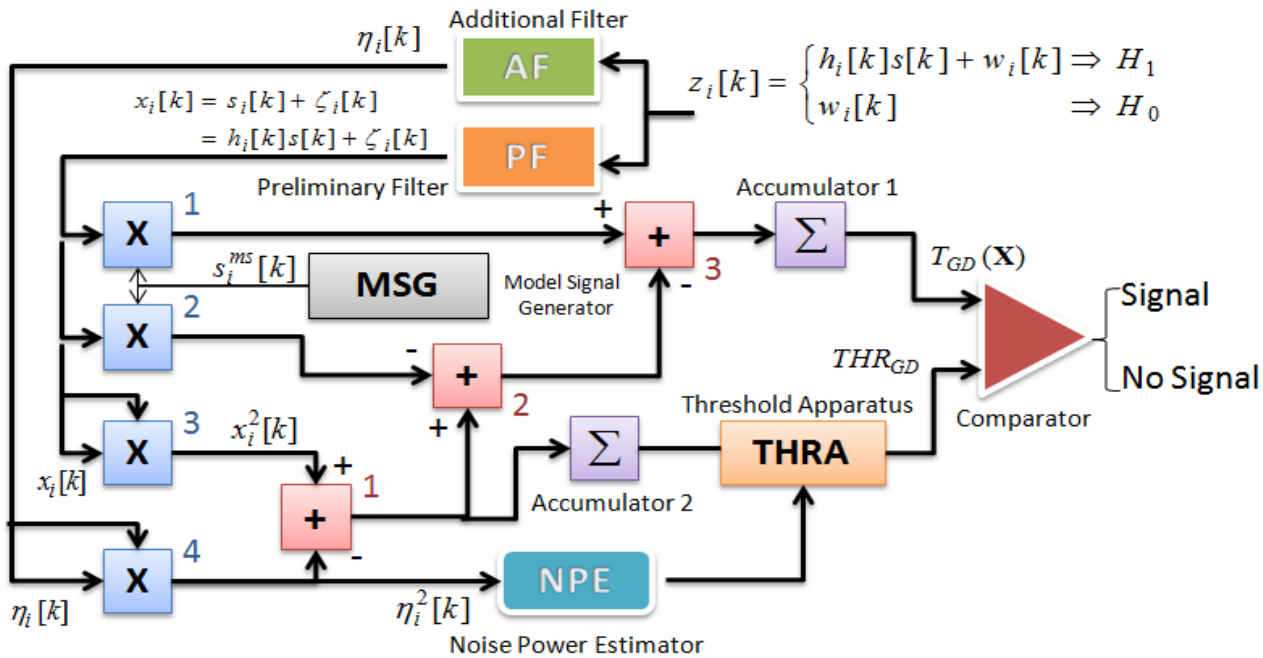

Fig. 1. Generalized receiver structure.

there is a need to employ the amplitude tracking system or to use the off-line data samples processing. Under the hypothesis Ft $_{0}$ ("a no" transmitted signal), satisfying the main GR functioning condition (2) at $\mu \rightarrow 1$ we obtain only the background noise $\eta_{i}^{2}[k]-\zeta_{i}^{2}[k]$ at the GR output.

Under practical implementation, the real structure of GR depends on specificity of signal processing systems and their applications, for example, the radar sensor systems, adaptive wireless communication systems, cognitive radio systems, satellite communication systems, and mobile communication systems and so on. In the present paper, the GR circuitry (Fig.1) is demonstrated with the purpose to explain the main functioning principles. Because of this, the GR flowchart presented in the paper should be considered under this viewpoint. Satisfying the GR main functioning condition (2) at $\mu \rightarrow 1$, the ideal case, for the wireless communication systems with radar sensor applications we are able to detect the transmitted signal with very high probability of detection and define accurately its parameters.

In the present paper, we discuss the GR implementation in communication systems using the radar sensor array. Since the presented GR test statistics is defined by the signal energy and noise power, the equality between the parameters of the model signal $s_{i}^{m}[k]$ and transmitted signal to be detected $s_{i}[k]$, in particular by amplitude, is required that leads us to high circuitry complexity in practice. For example, there is a need to employ the amplitude tracking system or off-line data sample processing. Detailed discussion about the main GR functioning principles if there is no a priori information and there is an uncertainty about the parameters of transmitted signal, i. e., the transmitted signal parameters are random, can be found in [14] and [15, Chapter 6, pp.611-621 and Chapter 7, pp. 631-695].
The complete matching between the model signal $s_{i}^{m}[k]$ and the incoming signal $s_{i}[k]$, for example by amplitude, is a very hard problem in practice because the incoming signal $s_{i}[k]$ depends on both the fading and the transmitted signal parameters and it is impractical to estimate the fading gain at the low SNR. This matching is possible in the ideal case only. The GD detection performance will be deteriorated under mismatching in parameters between the model signal $s_{i}^{m}[k]$ and the transmitted signal $s_{i}[k]$ and the impact of this problem is discussed in [21]-[27], where a complete analysis about the violation of the main GR functioning requirements is presented. The GR deci-sion statistics requires an estimation of the noise variance $\sigma_{\eta}^{2}$ using the reference noise $\eta_{i}[k]$ at the AF output.

Under the hypothesis $\mathscr{\not}_{1}$, the signal at the GR PF output, see Fig. 1, can be defined as

$$
x_{i}[k]=s_{i}[k]+\zeta_{i}[k] \text {, }
$$

where $\zeta_{i}[k]$ is the noise at the PF output and

$$
s_{i}[k]=h_{i}[k] s[k],
$$

where $h_{i}[k]$ are the channel coefficients. Under the hypothesis $\mathscr{H}_{0}$ and for all $i$ and $k$, the process $x_{i}[k]=\zeta_{i}[k]$ at the PF output is subjected to the complex Gaussian distribution and can be considered as the i.i.d. process.

In the ideal case, we can think that the signal at the GR AF output is the reference noise $\eta_{i}[k]$ with the same statistical parameters as the noise $\zeta_{i}[k]$. In practice, there is a difference between the statistical parameters of the noise $\eta_{i}[k]$ and $\zeta_{i}[k]$. How this difference impacts on the GR detection performance 
is discussed in detail in [20, Chapter 7, pp.63-695] and in [21] $-[24]$.

The decision statistics at the GR output presented in [14] and [16, Chapter 3] is extended for the case of antenna array when an adoption of multiple antennas and antenna arrays is effective to mitigate the negative attenuation and fading effects. The GR decision statistics can be presented in the following form:

$T_{G R}(\mathbf{X})=\sum_{k=0}^{N-1} \sum_{i=1}^{M} 2 x_{i}[k] s_{i}^{m}[k]$

$-\sum_{k=0}^{N-1} \sum_{i=1}^{M} x_{i}^{2}[k]+\sum_{k=0}^{N-1} \sum_{i=1}^{M} \eta_{i}^{2}[k] \underset{\mathscr{H}_{0}}{\underset{\mathcal{H}_{1}}{>}} T H R_{G R}$,

where

$$
\mathbf{X}=[\mathbf{x}(0), \ldots, \mathbf{x}(N-1)]
$$

is the vector of the random process at the GR PF output and $T H R_{G R}$ is the GR detection threshold.

Under the hypotheses $\mathscr{H}_{1}$ and $\mathscr{H}_{0}$ when the amplitude of the transmitted signal is equal to the amplitude of the model signal, $s_{i}^{m}[k]=s_{i}[k], \mu=1$, the GR decision statistics $T_{G D}(\mathbf{X})$ takes the following form in the statistical sense, respectively:

$$
\left\{\begin{array}{l}
\mathscr{H}_{1}: T_{G D}(\mathbf{X})=\sum_{k=0}^{N-1} \sum_{i=1}^{M} s_{i}^{2}[k]+\sum_{k=0}^{N-1} \sum_{i=1}^{M} \eta_{i}^{2}[k]-\sum_{k=0}^{N-1} \sum_{i=1}^{M} \zeta_{i}^{2}[k], \\
\mathscr{H}_{0}: T_{G D}(\mathbf{X})=\sum_{k=0}^{N-1} \sum_{i=1}^{M} \eta_{i}^{2}[k]-\sum_{k=0}^{N-1} \sum_{i=1}^{M} \zeta_{i}^{2}[k] .
\end{array}\right.
$$

In (7) the term $\sum_{k=0}^{N-1} \sum_{i=1}^{M} s_{i}^{2}[k]=E_{s}$ corresponds to the average transmitted signal energy, and the term $\sum_{k=0}^{N-1} \sum_{i=1}^{M} \eta_{i}^{2}[k]-$ $\sum_{k=0}^{N-1} \sum_{i=1}^{M} \zeta_{i}^{2}[k]$ is the background noise at the GR output. The GR output background noise is the difference between the noise power at the GR PF and GR AF outputs. Practical implementation of the GR decision statistics requires an estimation of the noise variance $\sigma_{\eta}^{2}$ using the reference noise $\eta_{i}[k]$ at the AF output.

\section{System Model for MIMO-MMSE}

Throughout the paper, the superscript $*$ denotes conjugation and transposition. Vectors and matrices are indicated by bold, | $\mathbf{A} \mid$ and det $\mathbf{A}$ denotes the determinant of matrix $\mathbf{A}$, and $\left\{a_{i, j}\right\}_{i, j=1, \ldots, N}$ is the $N \times N$ matrix with the elements $a_{i, j}, i, j=$ $1, \ldots, N$. The MIMO system investigated in this paper is characterized by $N_{T}$ transmitting and $N_{R}$ receiving antennas (see Fig.2).

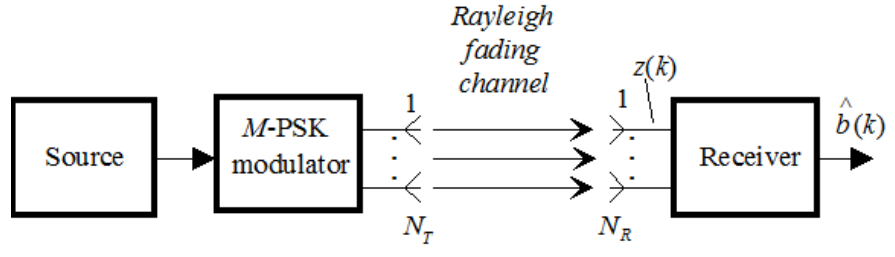

Fig. 2. Investigated MIMO system with $M$-PSK modulation.

The original data stream is divided in $N_{T}$ sub-streams, which are simultaneously transmitted by $N_{T}$ parallel $M$-PSK modulators. The $N_{R}$-dimensional signal $\mathbf{z}(k)$ at the output of the receiving antennas at discrete-time moment can be written in the following form

$\mathbf{z}(k)=\sqrt{E_{D}} \mathbf{C b}(k)+\mathbf{w}(k)$,

where $E_{D}$ is the mean (over fading) received energy of the signal transmitted by each antenna, $\mathbf{b}(k)$ accounts for the transmitted symbols with

$\left\{\begin{array}{l}\mathrm{E}\{\mathbf{b}(k)\}=0 ; \\ \mathrm{E}\left\{\mathbf{b}(k) \mathbf{b}^{*}(k)\right\}=\mathbf{I}\end{array}\right.$

$\mathbf{w}(k)$ is the additive Gaussian noise vector with

$\mathrm{E}\left\{\mathbf{w}(k) \mathbf{w}^{*}(k)\right\}=0.5 \mathcal{N}_{0}^{\rho} \mathbf{I}$

and $0.5 \mathcal{N}_{0}$ is the two-sided power spectral density of the thermal noise per antenna element. The matrix $\mathbf{C}$ is the $N_{R} \times N_{T}$ channel matrix

$\mathbf{C}=\left[\begin{array}{cccc}c_{11} & c_{12} & \cdots & c_{1 N_{T}} \\ c_{21} & c_{22} & \cdots & c_{2 N_{T}} \\ \vdots & \vdots & \cdots & \vdots \\ c_{N_{R} 1} & c_{N_{R} 2} & \cdots & c_{N_{R} N_{T}}\end{array}\right\rfloor$.

As in [1], [3]-[7], we consider slow frequency flat fading with the elements of the matrix $\mathbf{C}$, modeled as the independent identically distributed (i.i.d.) circular complex-valued Gaussian random variables having

$$
\left\{\begin{array}{l}
\mathrm{E}\left\{c_{i, j}\right\}=0 \\
\mathrm{E}\left\{\left|c_{i, j}\right|^{2}\right\}=1 .
\end{array}\right.
$$

In the MIMO system based on linear combining, the received vector $\mathbf{z}(k)$ is combined with the matrix $\mathbf{F}$ to obtain the decision variables

$\tilde{\mathbf{b}}(k)=\mathbf{F}^{*} \mathbf{z}(k)$.

The choice of $\mathbf{F}$ minimizing the expected square-error (MMSE criterion) between the transmitted symbols and the decision variables is given by the following well-known result [27]

$$
\mathbf{F}^{*}=\sqrt{E_{D}} \mathbf{B C}^{*}\left(E_{D} \mathbf{C B C} \mathbf{C}^{*}+\mathcal{N}_{0} \mathbf{I}\right)^{-1}
$$


where in our case we have $\mathbf{B}=\mathbf{I}$ based on the hypothesis of independence among the transmitted symbols. In this case Eq. (14) takes the form

$$
\mathbf{F}^{*}=\sqrt{E_{D}} \mathbf{R}^{-1} \mathbf{C}^{*},
$$

where the covariance matrix $\mathbf{R}$ is given by

$$
\mathbf{R}=\mathrm{E}\left\{\mathbf{z}(k) \mathbf{z}^{*}(k)\right\}=E_{D} \mathbf{C C}^{*}+0.5 \mathcal{N}_{0}^{\circ} \mathbf{I} .
$$

In the following, the MIMO systems with combining matrix $\mathbf{F}$ (15) have referenced to as the MIMO-MMSE.

After linear MMSE reception, the vector $\tilde{\mathbf{b}}(k)$ containing the linear MMSE estimates of the transmitted symbols is the vector $\mathbf{b}(k)$ further processed by a decision device to produce the estimated symbols $\tilde{\mathbf{b}}(k)$. In its simplest form, the decision device is composed of a bank of parallel devices, one for each component of $\tilde{\mathbf{b}}(k)$. This can be also interpreted as the vector linear equalizer, where the aim is to reduce the "intersymbol" interference (ISI) due to the parallel transmission of independent symbols over the nonorthogonal radio channel, rather than the ISI among symbols transmitted at different time epoch as in single channel systems. More sophisticated suboptimum strategies can be designed, including successive interference cancellation that acts in an analogous way to decision feedback equalizers, and will be investigated in Section V.

\section{Performance Evaluation of MIMO- MMSE System}

In this section, we will derive the SEP performance of MIMO-MMSE system constructed on the basis of the generalized receiver. The crux of the derivation is to observe that MIMO-MMSE reception is equivalent to a bank of parallel optimum combiners each with $N_{R}$ antennas estimating the signal transmitted by one of the antenna, and treating signals from other remaining $N_{T}-1$ antennas as interferers. This fact proven in Appendix I enable us to obtain the SEP performance of MIMO-MMSE system based on the generalized receiver by leveraging the analytical framework developed for optimum combining. The coherent detection of M-PSK modulated signals using the optimum combining with $N_{A}$ receiving antennas in the presence of $N_{I}$ equal-power interferers, each with the mean (over fast fading) received energy per symbol $E_{I}$, and the thermal noise in a flat Rayleigh-fading environment.

The SEP expressing of [13] requires the evaluation of nested $N_{\text {min }}$-fold integrals, with

$$
N_{\text {min }}=\min \left\{N_{R}, N_{T}-1\right\} \text {, }
$$

that it can be cumbersome for large $N_{\min }$. We extended this to reduce the computational complexity, as follows.

Now, there is a need to prove the following statement. The exact SEP expression for coherent detection of $M$-ary PSK with optimum combining, $N_{R}$ receive, and $N_{T}-1$ equal power co-channel interferers and thermal noise is
$S E P=\frac{K}{\pi} \int_{0}^{\Theta} A(\theta) \operatorname{det} \mathscr{E}(\theta) d \theta$

where

$$
A(\theta)=\left[\frac{\sin ^{2} \theta}{\sin ^{2} \theta+c_{M P S K} E_{D} / 2 \mathcal{N}_{0}}\right]^{N_{R}-N_{\min }},
$$

with $\Theta=\pi(M-1) / M$ and $c_{M P S K}=\sin ^{2}(\pi / M)$. The matrix $\mathscr{Q}(\theta)=\left\{G_{i+j}(\theta)_{i, j=0, \ldots N_{\min }-1}\right.$ is the Hankel matrix with elements given by

$$
\begin{aligned}
& G_{k-N_{\max }+N_{\min }}(\theta)=\varsigma(\theta)^{k} \exp \{\varsigma(\theta)\} k ! \\
& \times\left[\varsigma(\theta)(1+k) \Gamma(-1-k, \varsigma(\theta))+\left(2 \mathcal{N}_{0} / E_{I}\right) \Gamma(-k, \varsigma(\theta))\right],
\end{aligned}
$$

where

$$
\varsigma(\theta)=\frac{c_{M P S K} E_{D}}{E_{I} \sin ^{2} \theta}+\frac{2 \mathcal{N}_{0}}{E_{I}}
$$

$\Gamma(\alpha, z)$ is the incomplete Gamma function. Let us prove this statement.

The exact expression for the SEP of MIMO-MMSE system with the generalized receiver and $N_{A}=N_{R}$ receiving antennas and $N_{I}=N_{T}-1$ equal power co-channel interferers is given as

$S E P=\mathrm{E}_{\tilde{\lambda}}\left\{S E P_{\widetilde{\lambda}=\mathbf{x}}\right\}=\int_{0}^{\infty} \cdots \int_{0}^{\infty} \int_{0}^{\infty} S E P_{\tilde{\lambda}}(\mathbf{x}) f_{\widetilde{\lambda}}(\mathbf{x}) d x_{1} d x_{2} \cdots d x_{N_{\min }}$

Equation (22) was obtained in [13], [25], and [26] with the exception of different integration limits. This difference is due to the fact that unordered nonzero eigenvalues of the covariance matrix $\widetilde{\mathbf{R}}$ of [28]-[30] are used here in (22). In the present paper, the covariance matrix $\widetilde{\mathbf{R}}$ of [31], [32] is denoted by $\widetilde{\mathbf{R}}_{j}$ (Appendix I). In (22) we use the following notations: $S E P_{\widetilde{\lambda}=\mathbf{x}}$ is the symbol error probability conditioned on the given realizetion $\mathbf{x}=\left[x_{1}, \ldots, x_{N_{\min }}\right]^{T}$ of the $N_{\text {min }}$ nonzero unordered eigenvalues $\tilde{\lambda}=\left[\tilde{\lambda}_{1}, \ldots, \tilde{\lambda}_{N_{\min }}\right]^{T}$ of the central Wishart matrix (Appendix II), defined as

$\mathscr{W}\left(N_{\min }, N_{\max }\right)=\left\{\begin{array}{lll}\mathbf{C}_{j} \mathbf{C}_{j}^{*}, & \text { if } & N_{R} \leq N_{T}-1 ; \\ \mathbf{C}_{j}^{*} \mathbf{C}_{j}, & \text { if } & N_{R}>N_{T}-1,\end{array}\right.$

where $N_{\max }=\max \left\{N_{R}, N_{T}-1\right\} . S E P_{\tilde{\lambda}=\mathbf{x}}$ is given by [33]

$$
\operatorname{SEP}_{\bar{\lambda}}(\mathbf{x})=\frac{1}{\pi} \int_{0}^{\Theta} A(\theta) \prod_{i=1}^{N_{\min }}\left[\frac{\sin ^{2} \theta}{\sin ^{2} \theta+c_{M P S K} E_{D} /\left(E_{i} x_{i}+2 \mathcal{V}_{0}\right)}\right] d \theta
$$

and $f_{\tilde{\lambda}}(\mathbf{x})$ is the joint probability density function (pdf) of the random variable $\tilde{\lambda}$ given by (79), Appendix II. Substituting 
(79) in (22) we obtain

$$
\begin{aligned}
& S E P=\frac{K}{\pi N_{\min } !} \int_{0}^{\Theta} \int_{0}^{\infty} \ldots \int_{0}^{\infty} A(\theta)\left|\mathbf{V}_{1}(\mathbf{x})\right|^{2} \\
& \times \prod_{i=1}^{N_{\min }}\left[\frac{\sin ^{2} \theta \exp \left\{-x_{i}\right\} x_{i}^{N_{\max }-N_{\text {min }}}}{\sin ^{2} \theta+c_{M P S K} E_{D} /\left(E_{i} x_{i}+2 \mathcal{N}_{0}\right)}\right] d x_{1} d x_{2} \cdots d x_{N_{\text {min }}},
\end{aligned}
$$

where $\mathbf{V}_{1}(\mathbf{x})$ is the Vandermonde matrix (see Appendix II).The expression (25) can be simplified using the following statement, whose proof is given in [8]. Under the given two arbitrary $N \times N$ matrices $\boldsymbol{\Phi}(\mathbf{x})$ and $\boldsymbol{\Psi}(\mathbf{x})$ with $i$-th and $j$-th elements, i.e., $\Phi_{i}\left(x_{j}\right)$ and $\Psi_{i}\left(x_{j}\right)$, and an arbitrary function $\xi(\cdot)$ the following identity holds:

$$
\begin{aligned}
& \int \ldots \int_{D}|\boldsymbol{\Phi}(\mathbf{x})| \times|\boldsymbol{\Psi}(\mathbf{x})| \prod_{i=1}^{N} \xi\left(x_{i}\right) d \mathbf{x} \\
& =N ! \operatorname{det}\left\{\left.\int_{a}^{b} \Phi_{i}(x) \Psi_{j}(x) \xi(x) d x\right|_{i, j=1, \ldots, N}\right\},
\end{aligned}
$$

where the multiple integral is over the domain

$$
\mathcal{D}=\left\{a \leq x_{1} \leq b, a \leq x_{2} \leq b, \ldots, a \leq x_{N} \leq b\right\}
$$

and

$$
d \mathbf{x}=d x_{1} d x_{2} \cdots d x_{N} .
$$

Using (26) at

$$
\begin{aligned}
& a=0, b \rightarrow \infty, N=N_{\min }, \boldsymbol{\Phi}(\mathbf{x})=\mathbf{V}_{1}(\mathbf{x}), \mathbf{\Psi}(\mathbf{x})=\mathbf{V}_{1}(\mathbf{x}), \\
& \xi(x)=\frac{\sin ^{2} \theta \exp \{-x\} x^{N_{\max }-N_{\min }}}{\sin ^{2} \theta+c_{M P S K} E_{D} /\left(E_{i} x_{i}+2 \mathcal{N}_{0}\right)},
\end{aligned}
$$

we obtain

$$
\begin{aligned}
& S E P=\frac{K}{\pi} \\
& \times \int_{0}^{\Theta} \operatorname{det}\left|\int_{0}^{\infty} \frac{\left(x+2 \mathcal{N}_{0} / E_{I}\right) e^{-x} x^{N_{\max }-N_{\min }+i+j-2}}{x+2 \mathcal{N}_{0} / E_{I}+E_{D} c_{M P S K} / E_{I} \sin ^{2} \theta} d x\right|_{i, j=1, \ldots, N_{\min }} \mid d \theta .
\end{aligned}
$$

Finally, using the following identity

$$
\begin{aligned}
& \int_{0}^{\infty} \frac{\exp (-x) x^{n}(x+a)}{x+b} d x \\
& =b^{n} \exp (b) n ![b(1+n) \Gamma(-1-n, b)+a \Gamma(-n, b)]
\end{aligned}
$$

valid at $n>-1$ and $\arg \{b\} \neq \pi$, we obtain (18) that provides a concise SEP expression and is amenable for efficient evaluation involving only a single integral with finite limits of integration.

As shown in Appendix I, in the case of MIMO-MMSE system with the generalized receiver we can think of the linear MMSE combiner as equivalent to a bank of parallel optimum or MMSE combiners, each considering one of the signals transmitted by an antenna as the desired signal and the remaining
$N_{T}-1$ as interferers when $E_{D}=E_{I}$ due to the assumption of uniform mean power over the transmitting antennas.

When the decision device following the linear combiner is a bank of independent slicers, the SEP is the same for all layers and is given by (18) together with (19) and (20). In the following the SEP of the MIMO-MMSE systems with the generalized receiver and $N_{T}$ transmitting and $N_{R}$ receiving antennas is denoted as

$$
\left.\operatorname{SEP}_{M M S E}\left(N_{R}, N_{T}, E_{D} / 2 \mathcal{N}_{0}\right)\right]
$$

where $E_{D} / \mathscr{N}_{0}$ is the receive symbol $S N R$ per transmitting antenna. In the next section, the performance of various decision devices following the MMSE linear combiner is investigated.

\section{MIMO-MMSE System With SIC}

The practical generalized receiver structure suggested originally in [20] includes a linear combiner and successive interference cancellation. Although the linear MMSE combiner is expected to perform better than the zero-forcing combiner [3][5], the latter is usually investigated in the literature since it is easier to analyze. Here, we derive simple expressions for the performance of the MIMO systems based on the generalized receiver with the linear MMSE combiner followed by SIC and denoted as MIMO-MMSE-SIC systems based on the generalized receiver. We consider a low-complexity SIC algorithm in which one of the linear MMSE combiner outputs is chosen, and the corresponding transmitted symbol is estimated by a slicer. The contribution of the signal due to this detected symbol is then reconstructed and cancelled from the received vecor. The same procedure is replaced for all remaining symbols.

We note that the performance of the MIMO-MMSE-SIC system with the generalized receiver can be improved by a proper ordering of the symbols to be detected on the basis of the instantaneous channel state. The evaluation of its performance is beyond the scope of the present paper. It is well-known that a detection with decision feedback suffers from EP, that is, the cancellation of an erroneously detected symbol increases the power of the interfering terms and can cause significant performance degradation [34], [35]. The same phenomenon is present in MIMO receivers employing SIC. In the next subsections, we analyze the performance of the MIMO-MMSE-SIC system based on the generalized receiver for the cases of without EP (NEP) as well as with EP.

\subsection{Performance of MIMO-MMSE-SIC System without EP}

Equation (33) provides the starting point for evaluating the performance of the MIMO-MMSE-SIC system employing the generalized receiver with arbitrary choice of order in the symbol detection. Without loss of generality, in the following it will be assumed that in the $i$-th step we detect the $i$-th element $b_{i}(k)$ of $\mathbf{b}(k)$. It is easy to show that with SIC the SEP can be derived by using the following: 
$S E P_{M M S E-S I C}=\frac{1}{N_{T}} \sum_{i=1}^{N_{T}} S E P_{i}$,

where $S E P_{i}$ represents the probability of making an error in the detection of the $i$-th symbol. To derive $S E P_{i}$ let us define

$\mathbf{z}_{[i]}(k)$ as the received vector after the cancellation of the previously detected $(i-1)$ symbols, so that $\mathbf{z}_{[1]}(k)=\mathbf{z}(k)$. The index $k$ is omitted in the rest of the present paper for brevity. In the absence of EP we can write

$\mathbf{z}_{[2]}=\mathbf{z}-\sqrt{E_{D}} \mathbf{c}_{1} b_{1}=\sqrt{E_{D}} \sum_{i=2}^{N_{T}} \mathbf{c}_{i} b_{i}+\mathbf{w}$,

where $\mathbf{c}_{i}$ is the propagation vector corresponding to $b_{i}$. In general

$$
\mathbf{z}_{[i]}=\mathbf{z}-\sqrt{E_{D}} \mathbf{C}_{[i]} \mathbf{b}_{[i]}+\mathbf{w},
$$

where $\mathbf{b}_{[i]}$ is the vector of the remaining $N_{T}-i+1$ undetected symbols and $\mathbf{C}_{[i]}$ represents the $N_{R} \times\left(N_{T}-i+1\right)$ channel vectors corresponding to the $i-1$ estimated symbols. Equation (36) shows that $\mathbf{z}_{[i]}$ can be thought of as the received vector of the MIMO-MMSE system constructed based on the generalized receiver with $N_{R}$ receiving antennas and $N_{T}-i+1$ transmit antennas. Hence, $S E P_{i}$ is equal to $S E P_{M M S E}\left(N_{R}, N_{T}-i+1, E_{D} /\right.$ $\mathcal{N}_{0}$ ) and (34) becomes

$$
S E P_{M M S E-S I C}=\frac{1}{N_{T}} \sum_{i=1}^{N_{T}} S E P_{M M S E}\left(N_{R}, N_{T}-i+1, E_{D} / 2 \mathcal{N}_{0}\right),
$$

\subsection{Performance of MIMO-MMSE-SIC System with EP}

Note that (34) holds even in the presence of EP, provided that the probabilities $S E P_{i}$ take into account the effects of EP. Unfortunately, the definition of the exact expressions for $S E P_{i}$ is difficult. Here, we present a simple approach to estimate these probabilities, which are shown to be very accurate in the numerical results section.

By using the total probability theorem, we can write

$$
S E P_{i}=\sum_{j=0}^{N_{i}-1} P\left\{\text { error }_{i} \mid E_{j}^{(i)}\right\} P\left\{E_{j}^{(i)}\right\},
$$

where $N_{i}=2^{i-1}$ the mutually exclusive events $E_{j}^{(i)}$, with $P\left\{\bigcup E_{j}^{(i)}\right\}=1$, regarding the $i-1$ previous symbols decisions. $P\left\{\right.$ error $\left._{i} \mid E_{j}^{(i)}\right\}$ is the probability of making an error at the detection of the $i$-th symbol conditioned on the event $E_{j}^{(i)}$. Each event $E_{j}^{(i)}$ can be associated with the $(i-1)$ - dimensional vector $\mathbf{s}_{j}^{(i)}$, with the element $s_{j, m}^{(i)}$ equal to zero if the symbol at the step $m$ has been correctly detected, one otherwise. For example, $\mathbf{s}_{j}^{(i)}=[0,1,1,0, \ldots, 1]^{T}$ represents the event that the first symbol has been correctly detected, the second has been incorrectly detected, and so on. It is convenient for what follows to assume that $\mathbf{s}_{j}^{(i)}$ is the $(i-1)$ - dimensional vector containing the binary representation of the number $j$. To better understand our derivation of $P\left\{\right.$ error $\left._{i} \mid E_{j}^{(i)}\right\}$, let us consider a simple example with $i=2$. In this case, we have two sequences $\mathbf{s}_{0}^{(2)}=[0]$ and $\mathbf{s}_{1}^{(2)}=[1]$, associated to the events $E_{0}^{(2)}$ and $E_{1}^{(2)}$, respectively. $P\left\{\right.$ error $\left._{2} \mid E_{0}^{(2)}\right\}$ represents the error probability for the second symbol conditioned on the event that the first symbol has been correctly detected. Similarly $P\left\{\right.$ error $\left._{2} \mid E_{1}^{(2)}\right\}$ represents the error probability for the second symbol conditioned on the event that the first symbol has been erroneously detected. While $P\left\{\right.$ error $\left._{2} \mid E_{0}^{(2)}\right\}$ can be easily derived using the results of Section V-A, the evaluation of $P\left\{\right.$ error $\left._{2} \mid E_{1}^{(2)}\right\}$ is much more involved. To derive it, let us consider the received vector $\mathbf{z}_{[2]}$

$$
\begin{aligned}
& \mathbf{z}_{[2]}=\mathbf{z}-\sqrt{E_{D}} \mathbf{c}_{1} \hat{b}_{1}=\sqrt{E_{D}} \mathbf{c}_{1}\left(b_{1}-\hat{b_{1}}\right)+\sqrt{E_{D}} \sum_{i=2}^{N_{T}} \mathbf{c}_{i} b_{i}+\mathbf{w} \\
& =\sqrt{E_{D}} \mathbf{C}_{[2]} \mathbf{b}_{[2]}+\underbrace{\sqrt{E_{D}} \mathbf{c}_{1}\left(b_{1}-\hat{b_{1}}\right)}_{=\mathbf{w}_{\text {eq }}}+\mathbf{w},
\end{aligned}
$$

where $\hat{b}_{1}$ is the erroneous decision regarding $b_{1}$. The comparison of (35) and (39) shows that the error of the previous symbol results in an additional disturbance, which can cause severe performance degradation. To estimate this effect we approximate the term $\mathbf{w}_{e q}$ as the Gaussian random variable with the zero mean, i.e. $\mathrm{E}\left\{\mathbf{w}_{e q}\right\}=0$ and

$$
\mathrm{E}\left\{\mathbf{w}_{e q} \mathbf{w}_{e q}^{*}\right\}=\left(E_{D} \mathrm{E}\left\{\left|b_{1}-\hat{b}_{1}\right|^{2}\right\}+2 \mathcal{N}_{0}^{\circ}\right) \mathbf{I},
$$

where expectations are with respect to the thermal noise, symbols, and propagation vectors. It will be apparent in the Section VI that this Gaussian model is adequate for obtaining the SEP performance of MIMO systems. Under the high-SNR regime most of the errors will be such that $\hat{b}_{1}$ is one of the neighboring symbols of $b_{1}$. In this case,

$$
d_{\text {MPSK }} \cong\left|b_{1}-\hat{b}_{1}\right|^{2}=4 \sin ^{2}(\pi / M),
$$

and hence

$$
\mathrm{E}\left\{\mathbf{w}_{e q} \mathbf{w}_{e q}^{*}\right\}=\left(E_{D} d_{M P S K}+2 \mathcal{N}_{0}\right) \mathbf{I} .
$$

With this approximations, in $\mathbf{z}_{[2]}(39)$ is equivalent to the received vector of the MIMO-MMSE system based on the generalized detector with $N_{T}-1$ (since one symbol has been already detected) transmitting antennas each giving an equivalent $S N R$ per receiving antenna equal to 
$\frac{E_{D}}{E_{D} d_{M P S K}+2 \mathcal{N}_{0}}=\frac{1}{2 \mathcal{N}_{0} / E_{D}+d_{M P S K}}$

and, therefore

$P\left\{\right.$ error $\left._{2} \mid E_{1}^{(2)}\right\} \approx S E P_{M M S E}\left\{N_{R}, N_{T}-1, \frac{1}{2 \mathcal{N}_{0} / E_{D}+d_{M P S K}}\right\}$.

Equation (44) can be generalized for arbitrary $i$ and $E_{j}^{(i)}$. In fact, if the detection of the previous $i-1$ symbols has caused $n$ errors, the received vector $\mathbf{z}_{[i]}$ becomes

$$
\begin{aligned}
& \mathbf{z}_{[i]}=\sqrt{E_{D}} \mathbf{C}_{[i]} \mathbf{b}_{[i]}+\sqrt{E_{D}} \sum_{q \in\left\{\alpha_{h}\right\}} \mathbf{c}_{q}\left(b_{q}-\hat{b}_{q}\right)+\mathbf{w} \\
& =\sqrt{E_{D}} \mathbf{C}_{[i]} \mathbf{b}_{[i]}+\mathbf{w}_{e q},
\end{aligned}
$$

where $A_{n}$ denotes the set of indexes of the $n$ erroneously decided symbols. Since the transmitted symbols are statistically independent, we again have

$$
\left\{\begin{array}{l}
\mathrm{E}\left\{\mathbf{w}_{e q}\right\}=0 ; \\
\mathrm{E}\left\{\mathbf{w}_{e q} \mathbf{w}_{e q}^{*}\right\}=\left(n E_{D} d_{M P S K}+2 \mathcal{N}_{0}^{\circ}\right) \mathbf{I} .
\end{array}\right.
$$

Finally, the term $P\left\{\right.$ error $\left._{2} \mid E_{1}^{(2)}\right\}$ can be written in the following form

$$
\begin{aligned}
& P\left\{\text { error }_{i} \mid E_{j}^{(i)}\right\} \\
& \approx S E P_{M M S E}\left\{N_{R}, N_{T}-(i-1), \frac{1}{2 \mathcal{N}_{0} / E_{D}+n_{j}^{i-1} d_{M P S K}}\right\},
\end{aligned}
$$

where $n_{j}^{m}$ indicates the number of ones (wrong symbols) in the first $m$ positions of the vector $\mathbf{s}_{j}^{(i)}$.

Now, let us consider the evaluation of $P\left\{E_{j}^{(i)}\right\}$. This can be written as

$$
P\left\{E_{j}^{(i)}\right\}=P\left\{\mathbf{s}_{j}^{(i)}\right\}=P\left\{\bigcap_{n=1}^{i-1} s_{j, n}^{(i)}\right\} .
$$

By using the well-known relation

$$
P\left\{\bigcap_{n=1}^{i-1} \mathcal{A}_{n}\right\}=\prod_{n=1}^{N} P\left\{\mathcal{A}_{n} \mid \bigcap_{m=1}^{n-1} \mathcal{A}_{m}\right\}
$$

we obtain

$$
\begin{aligned}
& P\left\{\bigcap_{n=1}^{i-1} s_{j, n}^{(i)}\right\}=\prod_{n=1}^{i-1} P\left\{s_{j, n}^{(i)}, \bigcap_{m=1}^{i-1} s_{j, m}^{(i)}\right\}=P\left\{s_{j, 1}^{(i)}\right\} P\left\{s_{j, 2}^{(i)} \mid s_{j, 1}^{(i)}\right\} \cdots \\
& P\left\{s_{j, i-1}^{(i)}, \bigcap_{m=1}^{i-2} s_{j, m}^{(i)}\right\} .
\end{aligned}
$$

We now consider the term $P\left\{s_{j, i-1}^{(i)}, \bigcap_{m=1}^{i-2} s_{j, m}^{(i)}\right\}$. That represents the probability of error $s_{j, i-1}^{(i)}=1$ or a correct decision $s_{j, i-1}^{(i)}=0$ at the detection of the $(i-1)$-th symbol conditioned on the detection of the first $(i-2)$ symbols. This problem can be solved by means of the previous model used to evaluate the probability of error $P\left\{\right.$ error $\left._{i} \mid E_{j}^{(i)}\right\}$

$$
\begin{aligned}
& P\left\{s_{j, i-1}^{(i)}, \cap_{m=1}^{i-2} s_{j, m}^{(i)}\right\} \\
& 1-S E P_{M M S E}\left\{N_{R}, N_{T}-(i-2), \frac{1}{2 \mathcal{N}_{0} / E_{D}+\eta_{j}^{i-2} d_{M P S K}}\right\}, \\
& \text { if } s_{j, i-1}^{(i)}=0 ; \\
& \operatorname{SEP}_{M M S E}\left\{N_{R}, N_{T}-(i-2), \frac{1}{2 \mathcal{N}_{0}^{\circ} / E_{D}+\eta_{j}^{i-2} d_{M P S K}}\right\}, \\
& \text { if } s_{j, i-1}^{(i)}=1 .
\end{aligned}
$$

The particular structure of (51) allows us to write $P\left\{E_{j}^{(i)}\right\}$ as the product of conditioned $(i-1)$ probabilities, each conditioned on the result of the detection of the previous symbols that can be calculated by using an expression similar to (51)

$$
P\left\{E_{j}^{(i)}\right\} \approx \prod_{n=1}^{i-1} \mathscr{P}\left(N_{R}, N_{T}-1(n-1), \eta_{j}^{n-1}, s_{j, n}^{(i)}\right)
$$

where

$$
\mathscr{P}(x, e, z, w) \cong\left\{\begin{array}{c}
1-S_{M P P_{M S E}}\left[x, y, \frac{1}{2 \mathcal{N}_{0} / E_{D}+z d_{M P S K}}\right], \\
\text { if } w=0 \\
S E P_{M M S E}\left[x, y, \frac{1}{2 \mathcal{N}_{0} / E_{D}+z d_{M P S K}}\right], \\
\text { if } w=1 .
\end{array}\right.
$$

The final result of the previous analysis gives the performance of MIMO-MMSE-SIC reception with the following simple expression:

$$
\begin{aligned}
& S E P_{\text {MMSE-SIC }}=\frac{1}{N_{T}} \sum_{i=1}^{N_{T}} \sum_{j=0}^{N_{i}-1}\left\{\mathscr{P}\left(N_{R}, N_{T}-(i-1), \eta_{j}^{i-1}, 1\right)\right. \\
& \left.\times \prod_{n=1}^{i-1} \mathscr{P}\left(N_{R}, N_{T}-(i-1), \eta_{j}^{n-1}, s_{j, n}^{(i)}\right)\right\} .
\end{aligned}
$$

Note that for a given value of $E_{D} / 2 \mathcal{N}_{0}$ the number of terms $S E P_{M M S E}(\cdot)$ to be calculated in (54) depends only on $N_{T}$, and it is given by

$\sum_{j=1}^{N_{T}} j=0.5 N_{T}\left(N_{T}-1\right)$.

The error probability $\operatorname{SEP}_{M M S E}(\cdot)$ can be derived by using the exact formula (18) together with (19) and (20) or one of the approximate expressions given in [13]. 


\section{Numerical Results}

Here we demonstrate the comparison between the MIMOMMSE and MIMO-MMSE-SIC systems constructed on the basis of the generalized receiver using the analytical expressions obtained in previous sections. The performance is evaluated in terms of SEP plotted as a function of the total SNR per receiving antenna element and spectral efficiency $S_{\text {eff }}$, defined as

$$
\left\{\begin{array}{l}
S_{e f f}^{M I M O-M M S E}=\frac{N_{T} E_{D}}{2 \mathcal{N}_{0}} ; \\
S_{e f f}^{M I M O-M M S E-S I C}=N_{T} \times \log _{2} M
\end{array},\right.
$$

respectively. To assess the validity of the proposed approximate formulas, the performance of the MIMO-MMSE and MIMO-MMSE-SIC systems constructed on the basis of the generalized receiver is compared with bit-level simulations where over $10^{9}$ of symbols were generated.

The effect of EP on the performance of the MIMO-MMSESIC systems constructed on the basis of the generalized receiver is presented in Fig. 3 at $N_{R}=4$, quaternary phase shift keying (QPSK) modulation, and $N_{T}$ ranging from 2 to 4 . Using Fig. 3 we can see that the target $S E P$ is $10^{-3}$ and $N_{R}=N_{T}=4$, the hypothesis of absence of EP gives an optimistic estimate of the required $S N R$ by about $3 \mathrm{~dB}$.

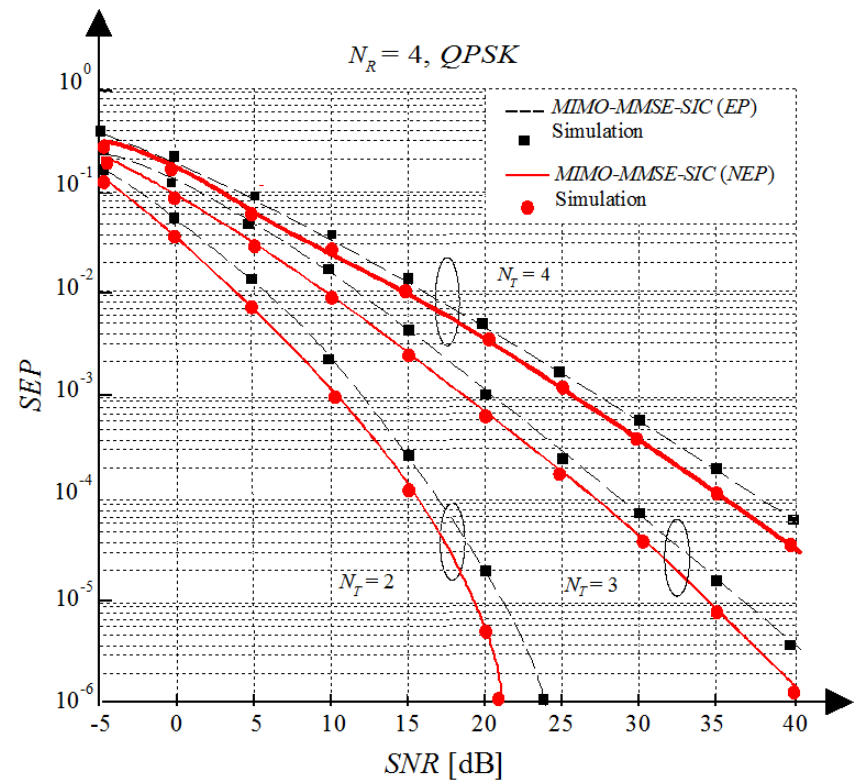

Fig. 3. Performance of MIMO-MMSE-SIC with EP and NEP for QPSK, $N_{R}=4$, and various values of $N_{T}=2 \div 4$.

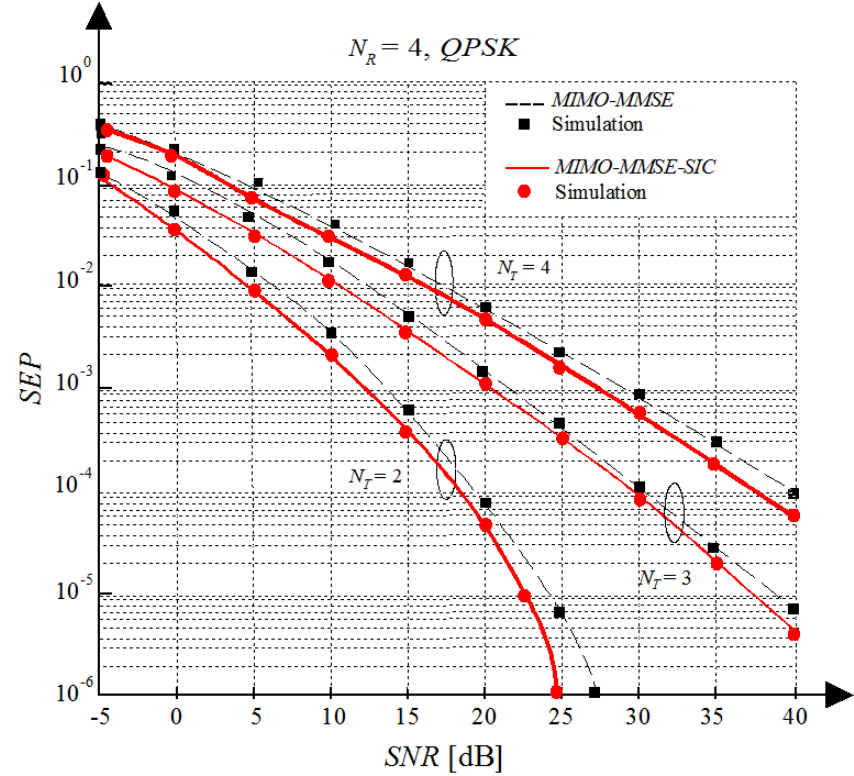

Fig. 4. Performance of MIMO-MMSE and MIMO-MMSESIC as a function of $S N R$ for QPSK, $N_{R}=4$, and various values of $N_{T}=2 \div 4$.

Furthermore, when small values of $N_{T}$ are considered, the number of cancellation steps decreases and the system is less sensitive to EP. In all subsequent figures, EP is taken into account. As clearly shown in Fig. 4, the MIMO-MMSE-SIC system constructed on the basis of the generalized receiver outperforms the MIMO-MMSE system constructed based on the generalized receiver. Moreover, the results confirm that our analytical results including the effects of EP are in excellent agreement with simulation results.

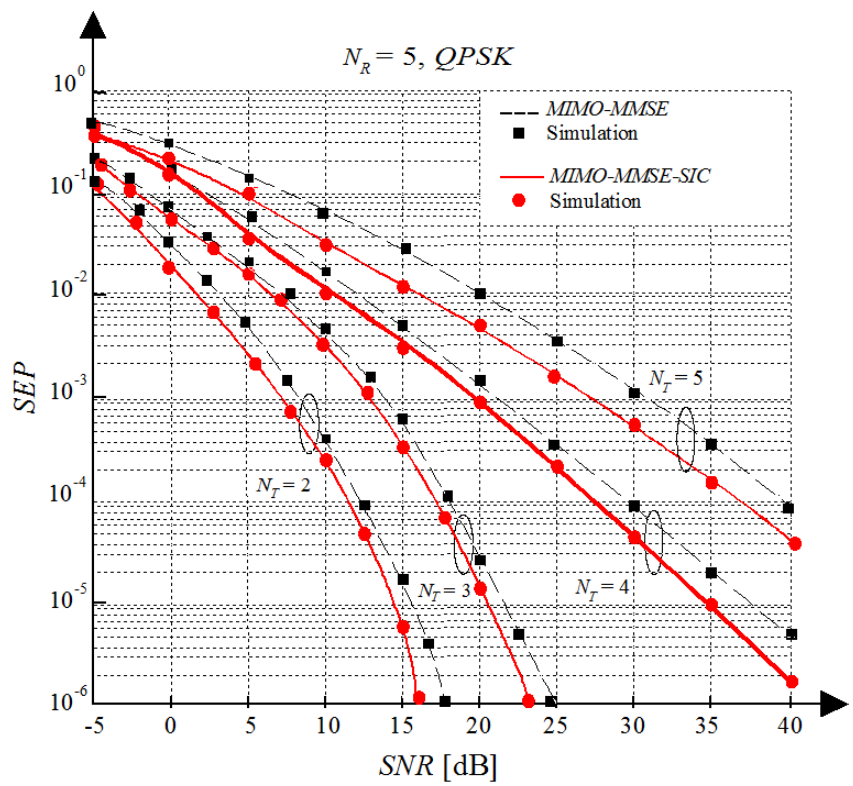

Fig. 5. Performance of MIMO-MMSE and MIMO-MMSESIC as a function of $S N R$ for QPSK, $N_{R}=5$, and various values of $N_{T}=2 \div 5$. 


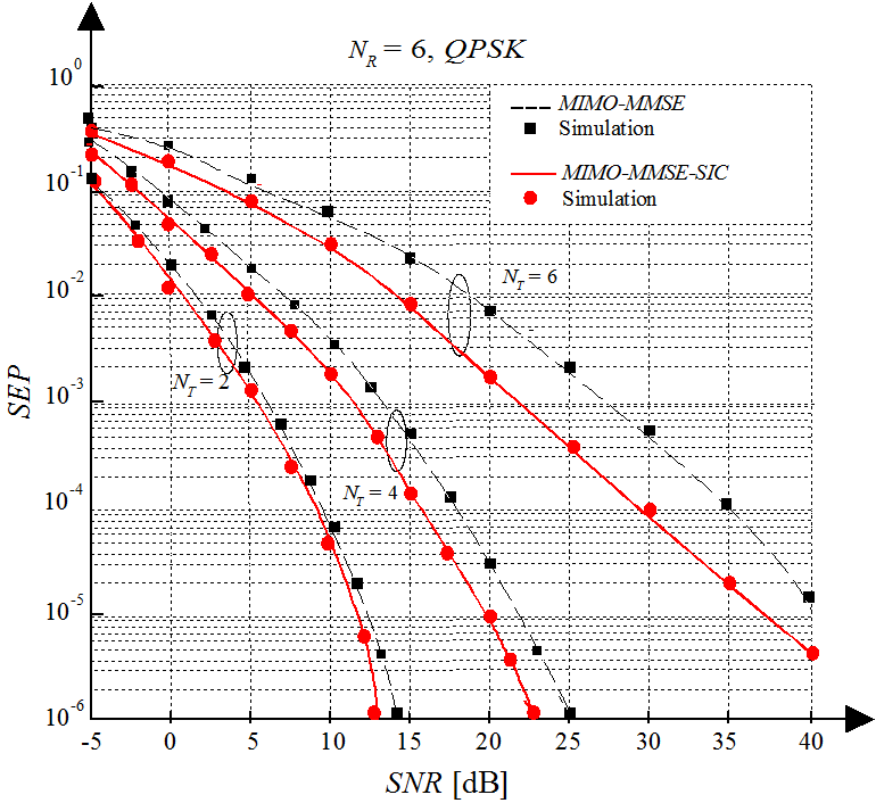

Fig. 6. Performance of MIMO-MMSE and MIMO-MMSESIC as a function of $S N R$ for QPSK, $N_{R}=6$, and various values of $N_{T}=2,4,6$.

The validity of the proposed model can also be appreciated in Figs. 5 and 6, where the same curves of Fig. 4 are shown but now $N_{R}$ equal to 5 and 6 , and different values of $N_{T}$ are considered. Note that using a number of receiving antennas larger than the number of transmitting antennas gives a large improvement due to the additional diversity gain available.

Figure 7 demonstrates the $S E P$ as a function of the number of transmitting antennas for $N_{R}=6$, QPSK modulation and different values of $S N R$. The figure clearly shows how, as expected, increasing in $N_{T}$ degrades the $S E P$ performance. On the other hand, increasing the number of transmit antennas provides an improvement in terms of spectral efficiency. In particular, if we fix the target $S E P$ at $10^{-3}$, we can achieve a spectral efficiency of $8 \mathrm{~b} / \mathrm{sec} / \mathrm{Hz}$ at $N_{T}=4$ for values of $S N R$ equal to $15 \mathrm{~dB}$ or higher.

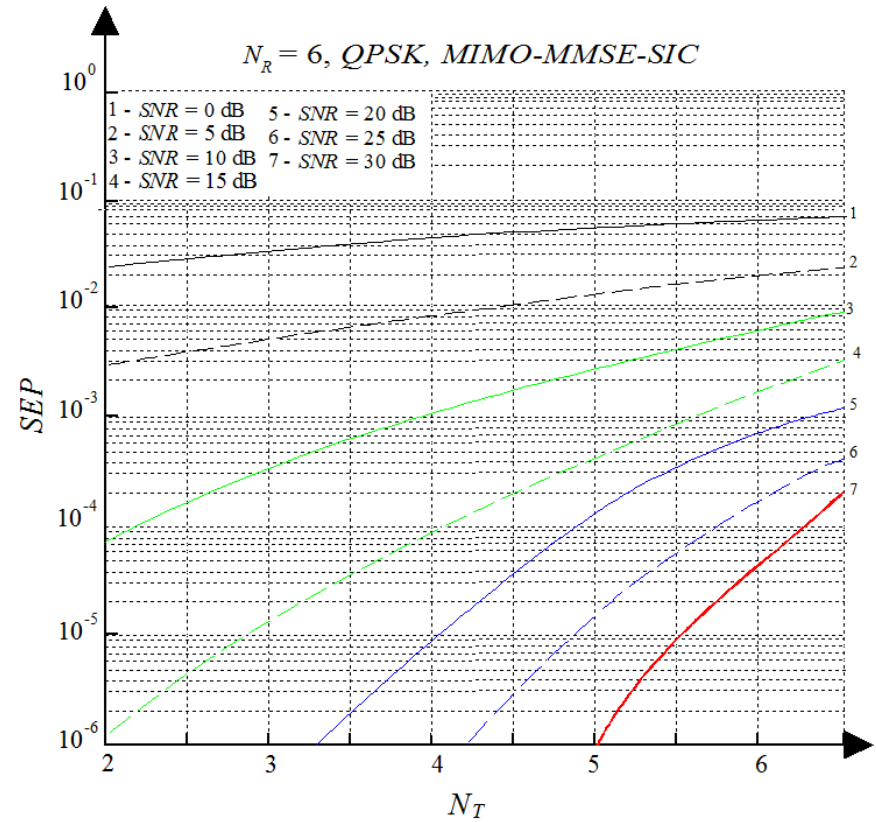

Fig. 7. Performance of MIMO-MMSE-SIC as a function of $N_{T}$ for QPSK, $N_{R}=6$, and various values of $S N R$ ranging from 0 to $30 \mathrm{~dB}$.

To appreciate the exceptional spectral efficiencies provided by these practical systems, we show in Fig. 8 the spectral efficiency of the MIMO-MMSE-SIC system constructed on the basis of the generalized receiver as a function of $S N R$ for different values of $N_{R}$ and $N_{T}$ at the target SEP equal to $10^{-3}$. The curves on Fig. 8, obtained from the analytical expressions developed in the previous sections and including EP, demonstrate that a spectral efficiency of $8 \mathrm{bit} / \mathrm{s} / \mathrm{Hz}$ can be achieved at the $S N R$ of about $29 \mathrm{~dB}$ with $N_{R}=4$ antennas. This value is reduced to about $15 \mathrm{~dB}$ at $N_{R}=6$.

Finally, Fig. 9 is similar to Fig. 8 but with reference to the bit-error probability $(B E P)$, here approximated as $S E P / \log _{2} M$ under the hypothesis of Gray coding [36], for different modulation formats, $N_{R}=6$ receiving antennas and the target $B E P$ of $10^{-3}$. Symbols on a given curve indicate the number of transmitting antennas $N_{T}=1 \div 6$. The crossing of the curves in Fig. 9 shows that for a given value of $S_{\text {eff }}$ there is an optimum modulation format with minimum required $S N R$. If we fix, for instance, $S_{\text {eff }}=16 \mathrm{bit} / \mathrm{s} / \mathrm{Hz}$, the minimal $S N R$ is achieved with 16-PSK and four transmitting antennas. The other modulation formats require a larger value of $S N R$ or provide the smaller spectral efficiency. 


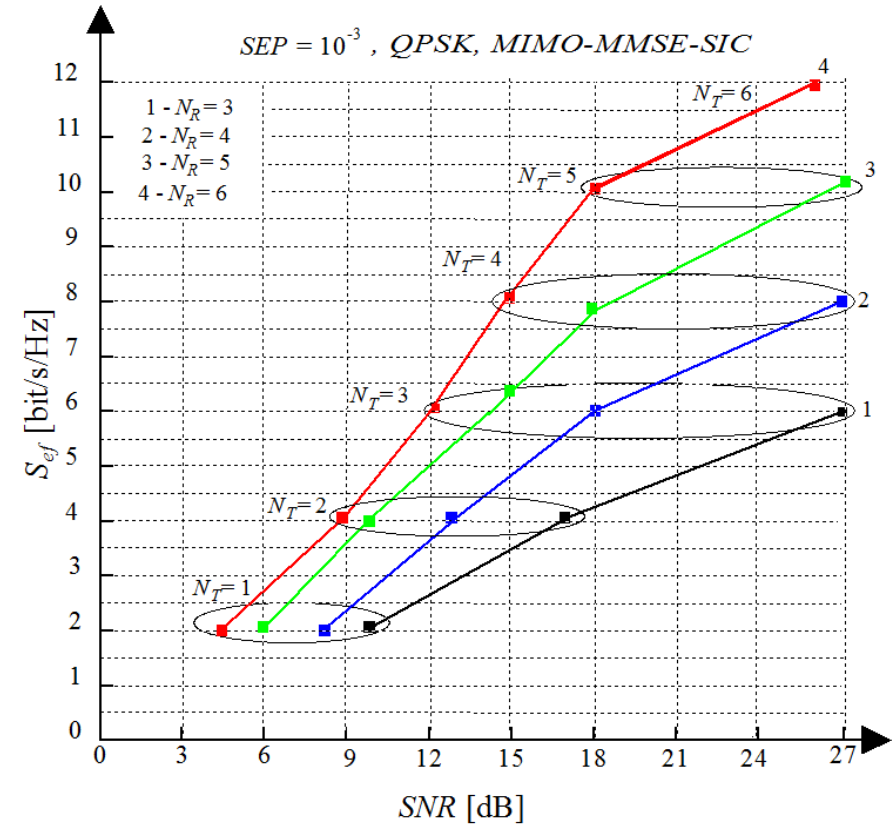

Fig. 8. Spectral efficiency of MIMO-MMSE-SIC as a function of $S N R$ for QPSK and various values of $N_{T}$ and $N_{R}$ at the target $S E P$ of $10^{-3}$.

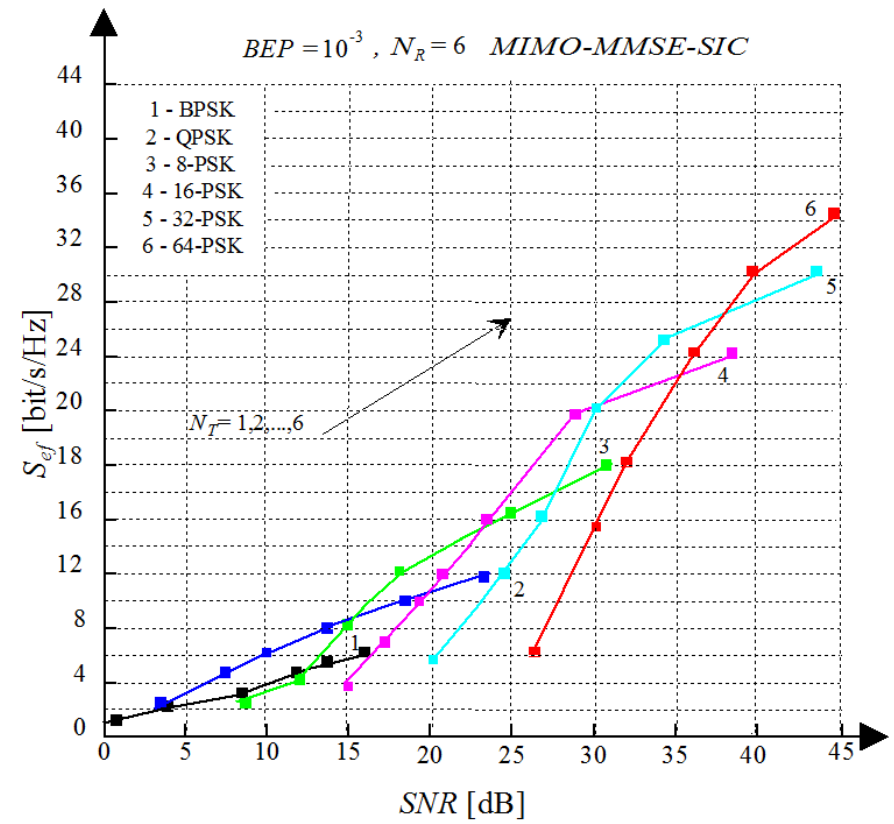

Fig. 9. Spectral efficiency of MIMO-MMSE-SIC as a function of $S N R$ for $N_{R}=6$ and various modulation format and $N_{T}$ at the target $B E P$ of $10^{-3}$.

\section{Conclusion}

In this paper, we have investigated the performance of high spectral efficiency of the MIMO systems based on the generalized receiver with $M$-PSK signals in a flat Rayleigh-fading environment. We first proposed a methodology to evaluate the $S E P$ for the MIMO systems with the generalized receiver based on the linear MMSE combining. Based on this methodology, we further derived the performance of the MIMO-MMSE system constructed on the basis of the generalized receiver followed by successive interference cancellation (MIMO-MMSE -SIC). We extended this to include the EP effect. Our results are valid for arbitrary number of transmitting and receiving antennas and are confirmed by Monte Carlo simulations.

\section{Appendix I}

\section{Equivalence Between Parallel Optimum and Joint Combining}

We note that the problem of detecting the $j$-th transmitted symbol in a MIMO system, in which case all others $N_{T}-1$ transmitted symbols can be thought of as interferers. Note that all the interfering signals are characterized by a man (over fast fading) received energy per symbol equal to $E_{D}$. This scenario is equivalent to a multiantenna system with $N_{A}=N_{R}$ receiving antennas and $N_{I}=N_{T}-1$ interfering signals, with the mean energy per symbol equal to $E_{I}=E_{D}$. It is well known that with optimal combining the weights that maximize the output signal-to-interference-plus-noise ratio (SINR) are given

$\mathbf{g}_{O C_{j}}=\varsigma_{j} \mathbf{R}_{j}^{-1} \mathbf{c}_{j}$,

where $\varsigma_{j}$ is the arbitrary constant that does not affect the array output $\operatorname{SINR}, \mathbf{c}_{j}$ is the propagation vector corresponding to $b_{j}$, and $\mathbf{R}_{j}$ is the covariance matrix given by

$$
\mathbf{R}_{j}=E_{D} \widetilde{\mathbf{R}}_{j}+2{ }_{e} \mathcal{N}_{0} \mathbf{I}
$$

with

$$
\widetilde{\mathbf{R}}_{j}=\mathbf{C}_{j} \mathbf{C}_{j}^{*},
$$

and

$$
\mathbf{C}_{j}=\left(\begin{array}{lllllll}
\mathbf{c}_{1} & \mathbf{c}_{2} & \cdots & \mathbf{c}_{j-1} & \mathbf{c}_{j+1} & \cdots & \mathbf{c}_{N_{T}}
\end{array}\right),
$$

where $\mathbf{c}_{j}$ is the column vector. Here we prove that (15) is equivalent to (57) and, therefore, the analytical framework developed in Section IV can be applied to investigate MIMO system with MMSE linear reception.

The $j$-th row of the matrix $\mathbf{G}^{*}$ in (5) that is given by

$\mathbf{g}_{j}^{*}=\sqrt{E_{D}} \mathbf{R}^{-1} \mathbf{c}_{j}^{*}$,

if we consider its conjugate transpose, we obtain

$\mathbf{g}_{j}=\sqrt{E_{D}} \mathbf{R}^{-1} \mathbf{c}_{j}$

where we used the property of the Hermitian matrices $\left(\mathbf{R}^{-1}\right)^{*}$ $=\mathbf{R}^{-1}$. To show that (62) is equivalent to $(57)$ we need to prove the following statement.

Let $\mathbf{D} \in M_{m, n}$ and $\mathbf{D}_{j} \in M_{m, n-1}$ be given by

$\mathbf{D}=\left(\begin{array}{llll}\mathbf{d}_{1} & \mathbf{d}_{2} & \cdots & \mathbf{d}_{n}\end{array}\right)$, 
and

$$
\mathbf{D}_{j}=\left(\begin{array}{lllllll}
\mathbf{d}_{1} & \mathbf{d}_{2} & \cdots & \mathbf{d}_{j-1} & \mathbf{d}_{j+1} & \cdots & \mathbf{d}_{n}
\end{array}\right),
$$

where $M_{m, n}$ denotes the set of the $(m \times n)$ complex matrices. Let $\mathbf{Q}$ and $\mathbf{Q}_{j}$ be equal to $K_{1} \mathbf{D D}^{*}+K_{2} \mathbf{I}$ and $K_{1} \mathbf{D}_{j} \mathbf{D}_{j}^{*}+K_{2} \mathbf{I}$, respectively, with scalars $K_{1} \geq 0$ and $K_{2} \geq 0$. Then, the complex vectors $\mathbf{v}_{j}=\mathbf{Q}^{-1} \mathbf{d}_{j}$ and $\mathbf{s}_{j}=\mathbf{Q}_{j}^{-1} \mathbf{d}_{j}$ are related as $\mathbf{v}_{j}=\gamma_{j} \mathbf{s}_{j}$ where

$$
\gamma_{j}=\frac{1}{1+K_{1} \mathbf{d}_{j}^{*} \mathbf{Q}_{j}^{-\mathbf{1}} \mathbf{d}_{j}},
$$

is a real nonnegative number.

Let us see the proof of this statement. Note that for any given $\mathbf{D}$, the square matrices $\mathbf{Q}$ and $\mathbf{Q}_{j}$ have the nonzero determinants and, hence, $\mathbf{Q}^{-1}$ and $\mathbf{Q}_{j}^{-1}$ exist. We can relate $\mathbf{v}_{j}$ and $\mathbf{s}_{j}$ as

$$
\mathbf{v}_{j}=\mathbf{Q}^{-1} \mathbf{p}_{j}=\mathbf{Q}^{-1}\left(\mathbf{Q}_{j} \mathbf{s}_{j}\right)
$$

Since that

$$
\mathbf{Q}=\mathbf{Q}_{j}+K_{1} \mathbf{d}_{j} \mathbf{d}_{j}^{*},
$$

we have

$$
\mathbf{v}_{j}=\mathbf{Q}^{-1}\left(\mathbf{Q}-K_{1} \mathbf{d}_{j} \mathbf{d}_{j}^{*}\right) \mathbf{s}_{j}=\mathbf{s}_{j}-\mathbf{v}_{j} K_{1} \mathbf{d}_{j}^{*} \mathbf{Q}_{j}^{-1} \mathbf{d}_{j},
$$

where we have used that fact that $\mathbf{v}_{j}=\mathbf{Q}^{-1} \mathbf{p}_{j}$ and $\mathbf{s}_{j}=\mathbf{Q}_{j}^{-1} \mathbf{p}_{j}$ Therefore,

$$
\mathbf{v}_{j}=\frac{\mathbf{s}_{j}}{1+K_{1} \mathbf{d}_{j}^{*} \mathbf{Q}_{j}^{-\mathbf{1}} \mathbf{d}_{j}}=\gamma_{j} \mathbf{s}_{j}
$$

and, hence, the vector $\mathbf{v}_{j}$ is proportional to $\mathbf{s}_{j}$. Since $\mathbf{Q}_{j}$ is positive definite, $\mathbf{d}_{j}^{*} \mathbf{Q}_{j}^{-1} \mathbf{d}_{j}>0$ which implies that the proportionality constant $\gamma_{j}$ is real positive.

If we now define

$$
\mathbf{D}=\mathbf{C}, \mathbf{D}_{j}=\mathbf{C}_{j}, K_{1}=E_{D}, K_{2}=2 \mathcal{N}_{0}
$$

Eq. (62) based on the statement that has been just proved can be written in the following form:

$$
\mathbf{g}_{j}=\frac{\sqrt{E_{D}} \mathbf{R}_{j}^{-1} \mathbf{c}_{j}}{1+E_{D} \mathbf{c}_{j}^{*} \mathbf{R}_{j}^{-1} \mathbf{c}_{j}}=\gamma_{j} \sqrt{E_{D}} \mathbf{R}^{-1} \mathbf{c}_{j} .
$$

Note that (71) is in the form (57). This fact establishes the equivalence between (15) and (57) and, therefore, all the results for optimum combining aiming to maximize the SINR can be used to investigate the MIMO-MMSE system constructed on the basis of the generalized receiver.

\section{Appendix II}

\section{Distribution of the Unordered Eigenvalues of a Wishart Matrix}

Let us define the $\left(N_{\min } \times N_{\max }\right)$, with $N_{\min } \leq N_{\max }$, complex matrix $\mathbf{A}$, with $\Sigma=\mathrm{E}\left\{\mathbf{a}_{j} \mathbf{a}_{j}^{*}\right\}, \forall j$ and $\left\{\left\{\mathbf{a}_{j} \mathbf{a}_{j}^{*}\right\}=0\right.$ for $i \neq$ $j$, where $\mathbf{a}_{j}$ is the $j$-th column vector of $\mathbf{A}$. If the elements of the matrix $\mathbf{A}$, i.e., $a_{i j}$ are the complex values with real and imaginary part each belonging to a normal distribution $N(0,1 / 2)$, then the Hermitian matrix

$$
\mathscr{W}\left(N_{\min }, N_{\max }\right)=\mathbf{A} \mathbf{A}^{*},
$$

is called the central Wishart. The distribution of the eigenvalues is studied in [37]. The joint pdf of the real ordered eigenvalues $\tilde{\lambda}_{1} \geq \tilde{\lambda}_{2} \geq \cdots \geq \tilde{\lambda}_{N_{\min }}$ takes the form

$$
\begin{aligned}
f_{\widetilde{\lambda}}\left(x_{1}, \ldots, x_{N_{\min }}\right)= & K|\boldsymbol{\Sigma}|^{-N_{\max }} 0 \widetilde{F}_{0}\left(-\boldsymbol{\Sigma}^{-1}, \mathbf{W}\right) \\
& \times|\mathbf{W}|^{N_{\text {max }}-N_{\min }} \prod_{i<j}^{N_{\min }}\left(x_{i}-x_{j}\right)^{2},
\end{aligned}
$$

where $K$ is a normalizing constant given by

$$
K=\frac{\pi^{N_{\min }\left(N_{\min }-1\right)}}{\widetilde{\Gamma}_{N_{\min }}\left(N_{\max }\right) \widetilde{\Gamma}_{N_{\min }}\left(N_{\min }\right)}
$$

with

$$
\widetilde{\Gamma}_{N_{\min }}(n)=\pi^{N_{\min }\left(N_{\min }-1\right) / 2} \prod_{i=1}^{N_{\min }}(n-i) !,
$$

${ }_{0} \widetilde{F}_{0}(\mathbf{A}, \mathbf{B})$ is known as the hypergeometric function of Hermitian matrix arguments, whose definition is given in $[31,(88)]$ in terms of series involving the zonal polynomials. These polynomials are, in general, very difficult to manage.

In the case of $\boldsymbol{\Sigma}=\mathbf{I}$, the joint pdf of the real ordered eigenvalues $\tilde{\lambda}_{1} \geq \tilde{\lambda}_{2} \geq \cdots \geq \tilde{\lambda}_{N_{\min }}$ of $\mathscr{W}\left(N_{\min }, N_{\max }\right)$ can be written as [31]

$$
f_{\widetilde{\lambda}}\left(x_{1}, \ldots, x_{N_{\min }}\right)=K \prod_{n=1}^{N_{\min }} \exp \left\{-x_{i}\right\} x_{i}^{N_{\max }-N_{\min }} \prod_{i<j}^{N_{\min }}\left(x_{i}-x_{j}\right)^{2},
$$

Denoting $\mathbf{x}=\left[x_{1}, x_{2}, \ldots, x_{N_{\min }}\right]$, the pdf in (76) can be written alternatively in terms of the Vandermonde matrix

$$
\mathbf{V}_{1}(\mathbf{x})=\left\{x_{j}^{i-1}\right\}_{i, j=1, \ldots, N_{\min }} .
$$

Since

$$
\left|\mathbf{V}_{1}(\mathbf{x})\right|^{2}=\prod_{i<j}^{N_{\min }}\left(x_{i}-x_{j}\right)^{2}
$$

equation (76) becomes

$$
f_{\widetilde{\lambda}}\left(x_{1}, \ldots, x_{N_{\text {min }}}\right)=\left|\mathbf{V}_{1}(\mathbf{x})\right|^{2} \prod_{n=1}^{N_{\text {min }}} \exp \left\{-x_{i}\right\} x_{i}^{N_{\max }-N_{\text {min }}} \text {. }
$$

Starting from (79), the joint pdf of the unordered eigenvalues 
of $\mathscr{W}\left(N_{\min }, N_{\max }\right)$ is easily written as

$$
\frac{K}{N_{\min } !}\left|\mathbf{V}_{1}(\mathbf{x})\right|^{2} \prod_{n=1}^{N_{\min }} \exp \left\{-x_{i}\right\} x_{i}^{N_{\max }-N_{\min }}
$$

\section{References}

[1] J. H. Winters, "On the capacity of radio communication systems with diversity in Rayleigh-fading environment." IEEE Journal of Selected Areas in Communications. 1987, Vol. SAC-5, No. 5. Pp. 871-878.

[2] M. Z. Win and J.H. Winters, "Virtual branch analysis of symbol error probability for hybrid selection/maximal-ratio combining in Rayleigh fading." IEEE Transactions on Communications. 2001, Vol. 49, No. 11, pp. 1926-1934.

[3] G. J. Foschini, "Layered space-time architecture for wireless communication in a fading environment when using multiple antennas." Bell Laboratory Technical Journal. 1996, Vol. 1, No. 2, pp. 41-59.

[4] G. J. Foschini and M. J. Gans, "On limits of wireless communications in a fading when using multiple antennas." Wireless Personal Communications. 1998. Vol. 6, No. 3, pp. 311-335.

[5] G. J. Foschini, G. D. Golden, R. A. Valenzuela, and P. W. Wolniansky, "Simplified processing for high spectral efficiency wireless communication employing multielement arrays," IEEE Transactions on Communications. 1999, Vol. 17, No. 11, pp. 1841-1852.

[6] E. Telatar, "Capacity of multiantenna Gaussian channels", European Transactions on Telecommunications. 1999, Vol. 10, No. 6, pp. 585-595.

[7] S. Loyka and G. Tsoulos, "Estimating MIMO system performance using the correlation matrix approach." IEEE Communications Letters. 2002, Vol. 6, No. 1, pp. 19-21.

[8] M. Chiani, M. Z. Win, and A., Zanella, "On the capacity of spatially correlated MIMO Rayleigh-fading channels." IEEE Transactions on Information Theory. 2003, Vol. 49, No. 10, pp. 2363-2371.

[9] S. Catreux, P. F. Driessen, and L. J. Grenstein, "Simulation results for an interference-limited multiple-input multiple-output cellular system." IEEE Communications Letters. 2000, Vol. 4, No. 11, pp. 334-336.

[10] N. Boubaker, K. B. Letaief, and D Murch, "Performance of BLAST over frequency-selective wireless communication channels." IEEE Transactions on Communications. 2002, Vol. 50, No. 2, pp. 196-199.

[11] A. Lozano and C. Papadias, "Layered space-time receivers for frequency-selective wireless channels." IEEE Transactions on Communications. 2002, Vol. 50, No. 1, pp. 65-73.

[12]X. Zhu and R. D. Murch, "Performance analysis of maximum likelihood detection in a MIMO antenna system." IEEE Transactions on Communications. 2002, Vol. 50, No. 2, pp. 187-191.

[13] M. Chiani, M. Z. Win, A Zanella, R. K. Mallik, and J. H. Winters, "Bounds and approximations for optimum combining of signals in the presence of multiple co-channel interferers and thermal noise." IEEE Transactions on Communications. 2002, Vol. 50, No. 1, pp. 65-73.
[14] V. P. Tuzlukov, "A new approach to signal detection theory." Digital Signal Processing. 1998, Vol. 8, No. 3, pp. 166-184.

[15] V.P. Tuzlukov, Signal Detection Theory, New York:Springer-Verlag, 2001.

[16] V.P. Tuzlukov, Signal Processing Noise, Boca Raton, London, New York, Washington D.C.: CRC Press, Taylor \& Francis Group, 2002.

[17] M. Maximov, "Joint correlation of fluctuative noise at the outputs of frequency filters. Radio Engineering. 1956, No. 9, pp. 28-38.

[18] Y. Chernyak, Y. "Joint correlation of noise voltage at outputs of amplifiers with no overlapping responses. Radio Physics and Electronics. 1960. No. 4, pp. 551-561.

[19] V. P. Tuzlukov, "DS-CDMA downlink systems with fading channel employing the generalized. Digital Signal Processing. 2011. Vol. 21, No. 6, pp. 725-733.

[20] V. P. Tuzlukov, "Signal processing by generalized detector in DS-CDMA wireless communication systems with frequency-selective channels." Circuits, Systems, and Signal Processing. Published on-line on February 2, 2011, doi:10.1007/s00034-011-9273-1; 2011, Vol.30, No.6, pp. 1197-1230.

[21] M. Shbat, Tuzlukov, V.P. "Primary signal detection algorithms for spectrum sensing at low SNR over fading channels in cognitive radio." Digital Signal Processing (2019) https://doi.org/10.1016/j.dsp. 2019.07.16. Digital Signal Processing. 2019, Vol. 93, No. 5, pp. 187- 207.

[22] M. Shbat, V. P. Tuzlukov, "Evaluation of detection performance under employment of the generalized detector in radar sensor systems." Radioengineering. 2014, Vol. 23, No. 1, pp. 50-65.

[23] M. Shbat, V. P. Tuzlukov, "Definition of adaptive detection threshold under employment of the generalized detector in radar sensor systems. IET Signal Processing.2014, Vol. 8, Issue 6, pp. 622-632.

[24] M. Shbat, V. P. Tuzlukov, SNR wall effect alleviation by generalized detector employment in cognitive radio networks. Sensors, 2015, 15(7), pp.16105-16135;doi:10. 3390/s150716105.

[25] V. P. Tuzlukov, "Quaternary DS-SSMA communications employing generalized detector over Rayleigh fading channels". WSEAS Transactions on Signal Processing. 2020. Vol.16., Paper \# 6, pp. 43-58. doi: 10. 37394/ 232014.2020.16.6

[26] V.P. Tuzlukov, "Signal processing by generalized receiver using DS-CDMA with STBC technology in multidimensional systems over fading channels: capacity and error probability." WSEAS Transactions on Communications. 2020. Vol. 19. Paper \#15. pp. 124-141. doi:10.37394/ 23204.2020.19.15

[27] R. N. McDonough and A. D. Whalen, Detection of Signals in Noise. $2^{\text {nd }}$ ed., San Diego, CA., USA: Academic Press, 1995.

[28] M. Jayasheela and A. Rajeswari,'Improved successive interference cancellation for $\mathrm{MIMO} / \mathrm{UWB}$-based wireless body area network", International Journal of Antennas and Propagation. Vol. 2012, Article ID 781434, 9 pages, 2012. https://doi.org/10.1155/2012/781434.

[29] P. Meerasri, P. Uthansakul, M. Uthansakul, "Self-interfe- 
rence cancellation- based mutual-coupling model for fullduplex single-channel MIMO systems". International Journal of Antennas and Propagation. Vol. 2014, Article ID 405487, 10 pages, 2014. https://doi.org/10.1155/2014/ 405487

[30] H. Na and C/ Lee, "An inter user interference cancellation scheme for two users in full-duplex MIMO system". EURASIP Journal on Wireless Communications and Networking. (2018) 2018: 171, 9 pages. https://doi.org/10. 1186/ s13638-018-1186-2

[31] Saifullah Adnan, Yuli Fu, Bhutto Jameed Ahmed, Muhammad Faizan Tahir, Farhad Banoon, "Modified ordered successive interference cancellation MIMO detection using low complexity constellation search"/AEU-International Journal of Electronics and Communications. 2020, Vol. 121, Article ID 153223. https://doi.org/10/1016/j. aeue.2020.153223

[32] R. Chatan and R. Akl, "Massive MIMO systems for $5 \mathrm{G}$ and beyond 32 networks-overview, recent trends, challenges,and future research direction". Sensors. 2020 May, 20 (10): 2753.doi:10.3390/s20102753

[33] Taehyoung Kim and Sangjoon Park, "Statistical beamforming for massive MIMO systems with distinct spatial correlations". Sensors. 2020 November, 20 (21): 6255. doi: $10.3390 / \mathrm{s} 20216255$.

[34] M. Chiani, "Introducing erasures in decision-feedback equalization to reduce error propagation." IEEE Transactions on Communications. 1997, Vol. 45, No. 7, pp. 757760.

[35] D. L. Duttweiler, J. E. Mazo, and G. Messerschmitt,” An upper bound on the error probability in decision-feedback equalizers." IEEE Transactions on Information Theory. 1974 Vol. IT- 20, No. 4, pp. 490-497.

[36] J. G. Proakis, Digital Communications. $4^{\text {th }}$ ed. New York: McGraw-Hill, 2008.

[37] A. T. James, "Distributions of matrix variates and latent roots derived from normal samples". Annual of Mathematical Statistics. 1964, Vol. 35, pp. 475-501

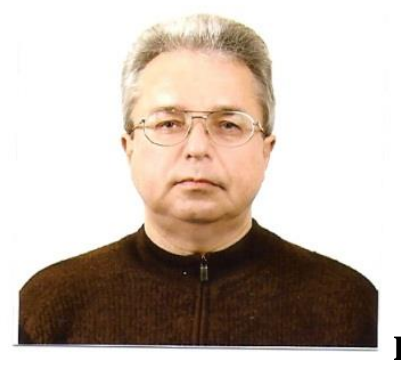

ed the MSc and $\mathrm{PhD}$ degrees in radio physics from the Belarusian State University, Minsk, Belarus in 1976 and 1990, respectively, and DSc degree in radio physics from the Kotelnikov Institute of Radioengineering and Electronics of Russian Academy of Sciences in 1995. Starting from 1995 and till 1998 Dr. Tuzlukov was a Visiting Professor at the University of San-Diego, San-Diego, California, USA. In 1998 Dr. Tuzlukov relocated to Adelaide, South Australia, where he served as a Visiting Professor at the University of Adelaide till 2000. From 2000 to 2002 he was a Visiting Professor at the
University of Aizu, Aizu-Wakamatsu City, Fukushima, Japan and from 2003 to 2007 served as an Invited Professor at the Ajou University, Suwon, South Korea, within the Department of Electrical and Computer Engineering. Starting from March 2008 to February 2009 he joined as a Full Professor at the Yeungnam University, Gyeonsang, South Korea within the School of Electronic Engineering, Communication Engineering, and Computer Science. Starting from March 1, 2009 Dr. Tuzlukov served as Full Professor and Director of Signal Processing Lab at the Department of Communication and Information Technologies, School of Electronics Engineering, College of IT Engineering, Kyungpook National University, Daegu, South Korea. Currently, Dr. Tuzlukov is the Head of Department of Technical Exploitation of Aviation and Radio Engineering Equipment, Belarusian State Academy of Aviation, Minsk, Belarus. His research emphasis is on signal processing in radar, wireless communications, wireless sensor networks, remote sensing, sonar, satellite communications, mobile communications, and other signal processing systems. He is the author over 300 journal and conference papers, eighteen books in signal processing published by Springer-Verlag and CRC Press. Some of them are Signal Detection Theory (2001), Signal Processing Noise (2002), Signal and Image Processing in Navigational Systems (2005), Signal Processing in Radar Systems (2012), Editor of the book Communication Systems: New Research (2013), Nova Science Publishers, Inc., USA, and has also contributed Chapters "Underwater Acoustical Signal Processing" and "Satellite Communications Systems: Applications" to Electrical Engineering Handbook: $3^{\text {rd }}$ Edition, 2005, CRC Press; "Generalized Approach to Signal Processing in Wireless Communications: The Main Aspects and Some Examples" to Wireless Communications and Networks: Recent Advances, InTech, 2012; "Radar Sensor Detectors for Vehicle Safety Systems" to Electrical and Hybrid Vehicles: Advanced Systems, Automotive Technologies, and Environmental and Social Implications, Nova Science Publishers, Inc., USA, 2014; "Wireless Communications: Generalized Approach to Signal Processing" and "Radio Resource Management and Femtocell Employment in LTE Networks", to Communication Systems: New Research, Nova Science Publishers, Inc., USA, 2013; "Radar Sensor Detectors for Vehicle Safety Systems" to Autonomous Vehicles: Intelligent Transport Systems and Automotive Technologies, Publishing House, University of Pitesti, Romania, 2013;"Signal Processing by Generalized Receiver in Wireless Communications Systems over Fading Channels," to Advances in Signal Processing: A Review, IFSA Publishing Corp. Barcelona, Spain. He participates as the General Chair, Keynote Speaker, Plenary Lecturer, Chair of Sessions, Tutorial Instructor and organizes Special Sections at the major International Conferences and Symposia on signal processing.

Dr. Tuzlukov was highly recommended by U.S. experts of Defence Research and Engineering (DDR\& E) of the United States Department of Defence as a recognized expert in the field of humanitarian demining and minefield sensing technologies and had been awarded by Special Prize of the United States Department of Defence in 1999. Dr. Tuzlukov is distinguished as one of the leading achievers from around the world by Marquis Who's Who and his name and biography have been 
included in the Who's Who in the World, 2006-2013; Who's Who in World, 25 ${ }^{\text {th }}$ Silver Anniversary Edition, 2008, Marquis Publisher, NJ, USA; Who's Who in Science and Engineering, 2006-2012 and Who's Who in Science and Engineering, 10th Anniversary Edition, 2008-2009, Marquis Publisher, NJ,USA; 2009-2010 Princeton Premier Business Leaders and Professionals Honours Edition, Princeton Premier Publisher, NY, USA; 2009 Strathmore's Who's Who Edition, Strathmore's Who's Who Publisher, NY, USA; 2009 Presidental Who's Who Edition, Presidental Who's Who Publisher, NY, USA; Who's Who among Executives and Professionals, 2010 Edition, Marquis Publisher, NJ, USA; Who's Who in Asia 2012, $2^{\text {nd }}$ Edition, Marquis Publisher, NJ, USA; Top 100 Executives of 2013 Magazine, Super Network Publisher, New York, USA, 2013; 2013/2014 Edition of the Global Professional Network, Business Network Publisher, New York, USA, 2013; 2013/2014 Edition of the Who's Who Network Online, Business Network Publisher, New York, USA, 2014; On-line Professional Gateway, 2014 Edition, Business Network Publisher, New York, USA, 2014; 2014 Worldwide Who's Who", Marquis Publisher, NJ, USA; 2015 Strathmore Professional Biographies, Strathmore's Who's Who Publisher, NY, USA; Who's Who in World, 2015, Marquis Publisher, NJ, USA; 2015-2016 Membership in Exclusive Top 100 network of professionals in the world, NY, USA, 2015; 2015 Who's Who of Executives and Professionals Honors Edition, Marquis Publisher, NJ, USA; Worldwide Who's Who - Top 100 Business Networking, San Diego, CA, USA, 2015.

Phone: +375173453283

Email: slava.tuzlukov@mail.ru

\section{Creative Commons Attribution License 4.0 (Attribution 4.0 International, CC BY 4.0)}

This article is published under the terms of the Creative Commons Attribution License 4.0

https://creativecommons.org/licenses/by/4.0/deed.en_US 